\title{
An extensive comparison of 0-1 linear programs for the daily satellite mission planning
}

\author{
Virginie Gabrel ${ }^{*}$
}

\begin{abstract}
Résumé
Dans ce papier, nous comparons plusieurs programmes linéaires en variables 0-1 pour résoudre le problème de la planification quotidienne des prises de vue à réaliser par un système d'observation de la Terre par satellite. Plusieurs heuristiques ont déjà été proposées pour résoudre ce problème combinatoire difficile. Afin d'évaluer la qualité des solutions approchées trouvées, il est utile de formuler le problème sous la forme d'un programme linéaire en variables 0-1. En effet, les valeurs des solutions optimales des relaxations continues fournissent des bornes supérieures de la valeur du problème. Dans ce papier, nous considérons deux modèles et nous expliquons pourquoi l'un des deux fournit nécessairement une meilleure borne. Notre démonstration se base sur les représentations du polytope de stable pour les graphes parfaits. Puis, nous calculons ces bornes sur des instances réalistes. Des améliorations encore possibles sont suggérées.
\end{abstract}

Mots-clefs : Optimisation combinatoire, polytope du stable, planification de missions spatiales, graphe de cocomparabilité

\begin{abstract}
In this paper, we compare several 0-1 linear programs for solving the satellite mission planning problem. Several heuristics have already been used to solve this difficult combinatorial problem. In order to assess the quality of the obtained approximate solutions, some 0-1 linear formulations have been proposed. Indeed, optimal solution values of linear relaxations provide upper bounds of the optimal solution value. In this paper, we consider two models and explain why one of both systematically compute lower upper bounds. Our explanation is based on stable set polytope formulations for perfect graphs. Then, we propose new upper bounds for some big size benchmark instances. Some improvement are also suggested.
\end{abstract}

Key words : Combinatorial optimization, stable set polytope, satellite mission planning, cocomparability graph

*LAMSADE, Université Paris-Dauphine, place du Maréchal de Lattre de Tassigny, 75775 Paris cedex 16, France. gabrel@lamsade.dauphine.fr 


\section{Introduction}

The mission planning problem of a low earth orbit satellite is the problem of choosing a set of photographs to be taken by the satellite during a planning horizon in order to satisfy a maximal part of the requested images. The mission planning problem of SPOT5 satellite has been the subject of numerous studies ([2], [3], [8], [4], [9]). Different approximate methods ([2], [8]) have been proposed and applied to the set of instances proposed in [3]. To assess the quality of the obtained approximate solutions, we need to compute tight upper bounds : in [2], the planning problem is formulated like a 0-1 linear program ; we have proposed in [4] another 0-1 linear formulation, using decomposition and flow formulation. In [9], a specific algorithm is proposed to compute very good upper bounds. In this paper, we explain why our 0-1 linear formulation presents a much smaller integrality gap than the formulation proposed in [2]. We compute upper bounds for all the instances proposed in [3]. These bounds are greater than those found in [9] but our results can easily be strengthened with some additional improvements.

\section{Different formulations for the daily satellite mission plan- ning problem}

\subsection{The daily satellite mission planning problem}

The SPOT satellite constitutes a family of Earth observation satellites, which are developped by the CNES (french Centre National d'Etudes Spatiales). SPOT5 has been launched in 2002. It is equipped with three viewing instruments (front, middle and rear) which can take two types of images : the mono image need one of the three instruments and the stereo image need the front and the rear instruments. The daily satellite mission planning problem of the SPOT5 satellite consists in deciding each day which image will be taken the next day in order to satisfy a maximal number of clients. The problem can be described as follows:

- The set of candidate images is known.

- A weight is associated with each image.

- The starting time and the completion time for taking each image is known.

- On an instrument, the main constraints concern the non-overlapping and the respect of a minimal transition time between two successive images.

- The total number of images is limited (due to limitation of the on-board recording capacity). 
- Some images cannot be taken simutaneously on different instruments (it is due to limitation of the instantaneous data flow through the satellite telemetry).

Each day, a set of images to be taken must be chosen in order to maximize the sum of the weights of selected images and to satisfy all constraints.

\subsection{The "natural" model}

In [2], a 0-1 linear program is proposed to modelize the daily mission planning problem of SPOT5. The model is quite natural: each image $i(i=1, \ldots, n)$ that can be taken by an instrument $c$ (with $c \in\{1,2,3\}$ ) is represented in the model by a 0 -1 variable $x_{i}^{c}$.

The proposed linear program, denoted $\mathrm{S} 5_{\mathrm{N}}$, is:

$$
\left(\mathrm{S} 5_{\mathrm{N}}\right) \begin{cases}\max \sum_{(i, c) \in S} w_{i} x_{i}^{c} & \\ \sum_{c:(i, c) \in S} x_{i}^{c} \leq 1 & \forall i \in M \\ x_{i}^{1}-x_{i}^{3}=0 & \forall i \in T \\ x_{i}^{c}+x_{j}^{c} \leq 1 & \forall\{(i, c),(j, c)\} \in \Omega \quad\left(1_{\mathrm{N}}\right) \\ \sum_{(i, c) \in \Lambda^{k}} x_{i}^{c} \leq \delta^{k} & k=1, \ldots, K \\ \sum_{(i, c) \in S} z_{i}^{c} x_{i}^{c} \leq z & \left(4_{\mathrm{N}}\right) \\ x_{i}^{c} \in\{0,1\} & \forall(i, c) \in S\end{cases}
$$

where

- $S$ denotes the set of all feasible couples $(i, c)$, and $w_{i}$ the weight of the image $i$,

- linear inequalities $\left(1_{\mathrm{N}}\right)$ correspond to the fact that each mono image $i$ ( $M$ denotes the mono set) must be taken by at most one camera,

- linear equalities $\left(2_{\mathrm{N}}\right)$ correspond to the fact that each stereo image $i$ ( $T$ denotes the stereo set) must either be simultaneously taken by the front camera (numbered by 1) and the rear one (numbered by 3 ) or not be taken.

- linear inequalities $\left(3_{\mathrm{N}}\right)$ correspond to constraints induced by non-overlapping and minimal transition time between two images taken on a same instrument, where $\Omega$ denotes the set of two couples $\{(i, c),(j, c)\}$ linked by these constraints.

- constraints $\left(4_{\mathrm{N}}\right)$ correspond to the constraints induced by limitation between several instruments, where $\Lambda^{k}$ denotes the set of two or three couples linked by constraint $k$ and $\delta^{k}$ equals to $\left|\Lambda^{k}\right|-1$, 
- constraint $\left(5_{\mathrm{N}}\right)$ corresponds the limitation on recording capacity where $z_{i}^{c}$ is the memory size necessary for recording on board image $i$ taken by camera $c$ and $z$ the total memory size available.

The optimal solution value of the linear relaxation of $\mathrm{S} 5_{\mathrm{N}}$, denoted $v\left(\overline{\mathrm{S}}_{\mathrm{N}}\right)$, is an obvious upper bound for $v\left(\mathrm{~S} 5_{\mathrm{N}}\right)$. But, as shown in section 4 , the integrality gap between $\mathrm{S} 5_{\mathrm{N}}$ and $\overline{\mathrm{S} 5_{\mathrm{N}}}$ is huge. Thus, we propose another strengthened 0-1 linear formulation.

\subsection{The flow formulation}

In [4], we propose another model based on a decomposition of the initial problem into three sub-problems, one per camera. Sub-problem on camera $c(c=1,2,3)$ concerns the choice of images to be taken by camera $c$ considering only constraints related to nonoverlapping and respect of a minimal transition time between two successive photographs. We denote $S^{c}$ the set of images that can be taken by camera $c$ and we define two binary relations on $S^{c} \times S^{c}$.

Definition 1 For $(i, j) \in S^{c} \times S^{c},(i, j)$ belongs to the incompatibility relation, denoted $\mathcal{I}^{c}$, if and only if $i$ and $j$ cannot be taken together because they use the camera $c$ at the same moment or the transition time between the two shots is not sufficient.

Let us remark that constraints $3_{\mathrm{N}}$ in $\mathrm{S} 5_{\mathrm{N}}$ describe the incompatibility relation on each set $S^{c}$.

Definition 2 For $(i, j) \in S^{c} \times S^{c},(i, j)$ belongs to the enchainability relation, denoted $\mathcal{E}^{c}$, if and only if $j$ is taken after $i$ with the instrument $c$.

The incompatibility relation is symmetric while the enchainability relation is clearly antisymmetric. Moreover, the enchainability relation is not necessarily transitive (it depends on satellite capacities).

Considering the enchainability relation, the sub-problem of choosing a set of images to be taken by an instrument $c$ can be represented by a directed graph, namely the enchainability graph denoted $G_{\mathcal{E}}^{c}=\left(V^{c}, E^{c}\right)$, defined as follows:

- $V^{c}$ represents the set $S^{c}$ (with $\left|S^{c}\right|=n^{c}$ ) of images that can be taken by camera $c$ (with the following convention: a couple $(i, c)$ is represented by vertex numbered by $i$ in $V^{c}$ ), plus two fictitious vertices numbered by 0 and $n^{c}+1$ respectively the root and the anti-root of $G_{\mathcal{E}}^{c}$,

- each vertex $i$ in $V^{c}$ is associated with the weight $w_{i}$ of image $i\left(w_{0}\right.$ and $w_{n^{c}+1}$ are equal to 0 ), 
- $E^{c}$ represents the enchainability relation defined on $S^{c} \times S^{c}$, plus arcs $(0, i)$ and $\left(i, n^{c}+1\right)$ for all $i=1, \ldots, n^{c}$, and $\left(0, n^{c}+1\right)$.

It appears that $G_{\mathcal{E}}^{c}$ is circuit-free since any arbitrary circuit $\left(i_{1}, i_{2}, \ldots, i_{q}, i_{1}\right)$ in $G_{\mathcal{E}}^{c}$ leads to the following contradiction: $i_{1}$ is taken before $i_{k}$ and $i_{k}$ is taken before $i_{1}$.

Any feasible sequence of images taken on camera $c$ can be represented by vertices belonging to a path $\mu\left[0, n^{c}+1\right]=\left\{i_{1}=0, i_{2}, \ldots, i_{q}=n^{c}+1\right\}$ from 0 to $n^{c}+1$ in $G_{\mathcal{E}}^{c}$. Respectively, any path $\mu$ from 0 to $n^{c}+1$ in $G_{\mathcal{E}}^{c}$ represents a sequence of images taken on camera $c$. This sequence respects all the constraints related to non-overlapping and respect of a minimal transition time between images if and only if $\mathcal{E}^{c}$ is transitive, otherwise some of these binary constraints may be violated. Indeed, if $\mathcal{E}^{c}$ is transitive, all vertices $i_{j}$ and $i_{j+k}$ belonging to $\mu$, with $k$ a strictly positive integer, are such that $\left(i_{j}, i_{j+k}\right) \in \mathcal{E}^{c}$. Otherwise, it may exist some vertices $i_{j}$ and $i_{j+k}$ belonging to $\mu$ (with $k \geq 2)$ such that $\left(i_{j}, i_{j+k}\right) \notin \mathcal{E}^{c}$. Consequently, associated constraints $x_{i_{j}}^{c}+x_{i_{j+k}}^{c} \leq 1$ are not respected by the sequence of images corresponding to $\mu$. In fact, the part of $3_{\mathrm{N}}$ constraints excluded from $G_{\mathcal{E}}^{c}$ are represented by arcs not in $E^{c}$ but belonging to the transitivity closure of $G_{\mathcal{E}}^{c}$, denoted $\hat{G}_{\mathcal{E}}^{c}=\left(V^{c}, \hat{E}^{c}\right)$ (obviously $\hat{E}^{c}=E^{c}$ when $G_{\mathcal{E}}^{c}$ is transitive).

So, the daily mission planning problem can be rewritten in terms of paths from 0 to $n^{c}+1$ in $G_{\mathcal{E}}^{c}$, with $c=1,2,3$, using the classical vertex-arc formulation as follows:

$$
\left(\begin{array}{l}
\max \sum_{i=1}^{n} \sum_{c=1}^{3} w_{i} \sum_{j \in \Gamma_{G_{\mathcal{E}}^{c}}(i)} \varphi_{i j}^{c} \\
\sum_{c=1}^{3} \sum_{j \in \Gamma_{G_{\mathcal{E}}^{c}}(i)} \varphi_{i j}^{c} \leq 1 \quad \forall i \in M \\
\sum_{j \in \Gamma_{G_{\mathcal{E}}^{1}}(i)} \varphi_{i j}^{1}-\sum_{j \in \Gamma_{G_{\mathcal{E}}^{3}}(i)} \varphi_{i j}^{3}=0 \quad \forall i \in T \\
A^{c} \varphi^{c}=b^{c} \forall c=1,2,3 \\
\sum_{j \in \Gamma_{G_{\mathcal{E}}^{c}}(i)} \varphi_{i j}^{c}+\sum_{k \in \Gamma_{G_{\mathcal{E}}^{c}}(j k)} \varphi_{j k}^{c} \leq 1 \forall(i, j) \in \hat{E}^{c} \backslash E^{c} \quad\left(3_{\mathrm{VA}}^{+}\right) \\
\sum_{(i, c) \in \Lambda^{k}} \sum_{l \in \Gamma_{G_{\mathcal{E}}^{c}}(i)} \varphi_{i l}^{c} \leq \delta^{k} \forall k=1, \ldots, K \\
\sum_{c=1}^{3} \sum_{i=1}^{n} z_{i}^{c} \sum_{j \in \Gamma_{G_{\mathcal{E}}^{c}}(i)} \varphi_{i j}^{c} \leq z \\
\varphi_{i j}^{c} \in\{0,1\} \forall(i, j) \in E^{c} \forall c=1,2,3
\end{array}\right.
$$


where each binary variable $\varphi_{i j}^{c}$ is associated with $\operatorname{arc}(i, j) \in E^{c}, \Gamma_{G_{\varepsilon}^{c}}(i)$ is the set of nodes adjacent to $i$ in $G_{\mathcal{E}}^{c}, A^{c}$ is the vertex-arc incidence matrix of $G_{\mathcal{E}}^{c}, b^{c}$ is an $n^{c}+2$ vector with all coefficients equal to 0 except the first equals to 1 and the last equals to -1 .

$\mathrm{S} 5_{\mathrm{VA}}$ is defined from $\mathrm{S} 5_{\mathrm{N}}$ by replacing variables $x_{i}^{c}$ by $\sum_{j \in \Gamma_{G_{\varepsilon}^{c}(i)}} \varphi_{i j}^{c}$ in all integer linear program excepted for $3_{N}$ constraints corresponding to arcs in $E^{c}$. Constraints $3_{N}$ corresponding to arcs in $E^{c}$ are translated in terms of paths with constraints $3_{\mathrm{VA}}$.

The optimal solution value of the linear relaxation of $\mathrm{S} 5_{\mathrm{VA}}$, denoted $v\left(\overline{\mathrm{S} 5} 5_{\mathrm{VA}}\right)$, is an upper bound for $v\left(\mathrm{~S} 5_{\mathrm{VA}}\right)$ equals to $v\left(\mathrm{~S}_{\mathrm{N}}\right)$. In the next section, we show that $v\left(\overline{\mathrm{S} 55_{\mathrm{VA}}}\right) \leq$ $v\left(\overline{\mathrm{S} 5_{\mathrm{N}}}\right)$.

\section{Models comparison}

In [8], the daily mission planning problem is seen as a knapsack problem with a huge number of additional logic constraints on variables. In order to compare $\overline{\mathrm{S} 5} 5_{\mathrm{VA}}$ and $\overline{\mathrm{S} 5_{\mathrm{N}}}$, we prefer to consider this problem as a maximum weighted stable set problem with additional linear constraints. Indeed, on each camera $c$, a set of images that can be taken together can be represented by a stable set in a graph, namely the incompatibility graph, denoted $G_{\mathcal{I}}^{c}=\left(X^{c}, I^{c}\right)$, defined as follows:

- $X^{c}$ represents the set $S^{c}$ (with $\left|S^{c}\right|=n^{c}$ ) of images that can be taken by camera $c$ (with the same convention: a couple $(i, c)$ is represented by vertex numbered by $i$ in $X^{c}$ ),

- each vertex $i$ in $X^{c}$ is associated with the weight $w_{i}$ of image $i$

- $I^{c}$ represents the incompatibility relation defined on $S^{c} \times S^{c}$.

Any set of images that can be taken on camera $c$ corresponds to a set of vertices belonging to a stable set in $G_{\mathcal{I}}^{c}$. Respectively, any stable set in $G_{\mathcal{I}}^{c}$ represents a set of images that can be taken on camera $c$. In the following section, we recall some wellknown results about stable set polytope formulations.

\subsection{About the stable set polytope}

Let us consider the stable set problem in an arbitrary graph $G=(V, E)$. The stable set polytope, usually denoted $\operatorname{StAB}(G)$ can be written that way:

$$
\operatorname{STAB}(G)=\operatorname{conv}\left(x^{S} \in\{0,1\}^{|V|}: S \subseteq V \text { is a stable set in } G\right) .
$$


where $x^{S}$ is the incidence vector of $S$.

The stable set polytope and its LP-relaxation has been the subject of numerous studies (see i.e [6]).

The edge constraints of the form

$$
x_{i}+x_{j} \leq 1 \forall(i j) \in E
$$

are facet-defining inequalities of $\operatorname{STAB}(G)$. And we have:

$$
\operatorname{STAB}(G)=\operatorname{conv}\left(x \in\{0,1\}^{|V|}: x \text { satisfies all edge constraints }\right)
$$

In general cases, the edge polytope defined as follows

$$
\operatorname{ESTAB}(G)=\left\{x \in \mathbb{R}_{+}^{|V|}: x \leq 1 \text { and satisfies all edge constraints }\right\}
$$

is not equal to the stable set polytope (except for bipartite graphs, see i.e [6]). In other words, all edge constraints are not sufficient to describe the stable set polytope, additional facet-defining inequalities must be introduced.

Obviously, maximal clique constraints of that form

$$
\sum_{i \in Q} x_{i} \leq 1, \text { with } Q \subseteq V
$$

where $Q$ is a maximal clique in $G$, generalize edge constraints. The clique polytope (also called fractional stable set polytope) is defined as follows

$$
\operatorname{QSTAB}(G)=\left\{x \in \mathbb{R}_{+}^{|V|}: x \text { satisfies all maximal clique constraints }\right\}
$$

For arbitrary graph, we have $\operatorname{STAB}(G) \subseteq \mathrm{QSTAB}(G) \subseteq \operatorname{ESTAB}(G)$. But, if $G$ is a perfect graph, it appears that $\operatorname{STAB}(G)=\operatorname{QSTAB}(G)$.

\subsection{Stable set polytope and daily mission planning problem formula- tions}

For a given $c$, constraints $x_{i}^{c}+x_{j}^{c} \leq 1 \forall(i, j) \in I^{c}$ are edge constraints on $G_{\mathcal{I}}^{c}=\left(X^{c}, I^{c}\right)$ and, the polytope conv $\left\{x^{c} \in\{0,1\}^{n^{c}}: x_{i}^{c}+x_{j}^{c} \leq 1 \forall(i, j) \in I^{c}\right\}$ defines the stable set polytope $\operatorname{STAB}\left(G_{\mathcal{I}}^{c}\right)$. Consequently, in $\overline{\mathrm{S} 5_{\mathrm{N}}}$, this stable set polytope is replaced by the corresponding edge polytope $\operatorname{ESTAB}\left(G_{\mathcal{I}}^{c}\right)=\left\{x^{c} \in[0,1]^{n^{c}}: x_{i}^{c}+x_{j}^{c} \leq 1 \forall\{(i, j)\} \in \mathcal{I}^{c}\right\}$ which does not correspond to $\operatorname{STAB}\left(G_{\mathcal{I}}^{c}\right)$ since there is no reason for $G_{\mathcal{I}}^{c}$ to be a bipartite graph. This difference between the two polytopes partially explains the huge integrality gap between $\mathrm{S} 5_{\mathrm{N}}$ and $\overline{\mathrm{S} 5_{\mathrm{N}}}$. In fact, the difference between $\overline{\mathrm{S} 5_{\mathrm{N}}}$ and $\overline{\mathrm{S} 5_{\mathrm{VA}}}$ is related to $\operatorname{STAB}\left(G_{\mathcal{I}}^{c}\right)$ formulations. 
Theorem $1 v\left(\overline{\mathrm{S} 5 \overline{V A}_{\mathrm{VA}}}\right) \leq v\left(\overline{\mathrm{S} 5_{\mathrm{N}}}\right)$.

Proof 1 Let us first suppose the transitivity of the enchainability relation. In this case, any stable set in $G_{\mathcal{I}}^{c}$ corresponds to a path from 0 to $n^{c}+1$ in $G_{\mathcal{E}}^{c}$ and conversely. In fact, $G_{\mathcal{E}}^{c}$ can be easily deduced from $G_{\mathcal{I}}^{c}$ as follows: define the complementary graph of $G_{\mathcal{I}}^{c}$, replace each edge between $i$ and $j$ by an arc from $i$ to $j$ if image $i$ is taken before image $j$, and by an arc from $j$ to $i$ otherwise. The obtained directed graph is exactly $G_{\mathcal{E}}^{c}$. Let us remark that a graph such that its complementary graph has a transitive orientation is a well-known perfect graph, namely cocomparability graph. In [1], it is shown that stable set polytope in cocomparability graph can be exactly described by the path-based formulation. Consequently, the polytope $\Phi=\left\{\varphi_{i j}^{c} \in[0,1]^{\left|E^{c}\right|}: A^{c} \varphi^{c}=b^{c}\right\}$ is equal to $\operatorname{STAB}\left(G_{\mathcal{I}}^{c}\right)$ for all $c=\{1,2,3\}$, and included in the edge polytope $\operatorname{ESTAB}\left(G_{\mathcal{I}}^{c}\right)$. Thus, $v\left(\overline{\mathrm{S} 5} 5_{\mathrm{VA}}\right) \leq v\left(\overline{\mathrm{S} 5_{\mathrm{N}}}\right)$.

In the non transitivity case, any path from 0 to $n^{c}+1$ in $G_{\mathcal{E}}^{c}$ corresponds to a stable set in a subgraph $\tilde{G}_{\mathcal{I}}^{c}$ of $G_{\mathcal{I}}^{c}$. $\tilde{G}_{\mathcal{I}}^{c}$ is obtained from $G_{\mathcal{I}}^{c}$ by removing all edges $(i, j)$ such that either $(i, j)$ or $(j, i)$ belongs to $\hat{E}^{c} \backslash E^{c}$. $\tilde{G}_{\mathcal{I}}^{c}$ is a cocomparability graph and the polytope $\Phi$ corresponds to the stable set polytope of $\operatorname{STAB}\left(\tilde{G}_{\mathcal{I}}^{c}\right)$ which is included in $\operatorname{ESTAB}\left(\tilde{G}_{\mathcal{I}}^{c}\right)$. Since $\operatorname{STAB}\left(G_{\mathcal{I}}^{c}\right)=\operatorname{STAB}\left(G_{\mathcal{I}}^{c} \backslash \tilde{G}_{\mathcal{I}}^{c}\right) \cap \operatorname{STAB}\left(\tilde{G}_{\mathcal{I}}^{c}\right)$ and, in $\overline{\operatorname{S5}} 5_{\mathrm{N}}, \operatorname{STAB}\left(\tilde{G}_{\mathcal{I}}^{c}\right)$ is replaced by $\operatorname{ESTAB}\left(\tilde{G}_{\mathcal{I}}^{c}\right)$ while, in $\overline{\mathrm{S} 5_{\mathrm{VA}}}, \operatorname{STAB}\left(\tilde{G}_{\mathcal{I}}^{c}\right)$ is exactly described by $\Phi$, we have $v\left(\overline{\mathrm{S} 5_{\mathrm{VA}}}\right) \leq v\left(\overline{\mathrm{S} 5_{\mathrm{N}}}\right)$.

We compute $v\left(\overline{\mathrm{S} 5_{\mathrm{N}}}\right)$ and $v\left(\overline{\mathrm{S} 5_{\mathrm{VA}}}\right)$ on a set of realistic benchmark instances. Results are presented in the following section.

\section{Experimentations and results}

Experiments are carried out on a set of realistics instances described in [3]. There exists two classes of instances:

- the first class includes small size instances without recording capacity constraint,

- the second class includes bigger size instances with recording capacity constraint.

For the first class instances, optimal solution values are known. For the second class instances, approximate solutions have been determining using tabu search (for details see [8]) and, for some of them, optimality has been proved in [9].

Each instance is only described by a list of all binary and ternary constraints. So, when two images $i$ and $j$ are not linked by a constraint, we do not know if $i$ is taken before $j$ 


\begin{tabular}{c|c|c|c|c|c|c} 
Instance & $\begin{array}{c}\text { number of } \\
\text { images }\end{array}$ & $v\left(\mathrm{~S} 5_{\mathrm{N}}\right)$ & $v\left(\overline{\mathrm{S} 5_{\mathrm{N}}}\right)$ & $v\left(\overline{\mathrm{S} 5} 5_{\mathrm{VA}}\right)$ & $\frac{v\left(\overline{\mathrm{S} 5_{\mathrm{N}}}\right)-v\left(\mathrm{~S} 5_{\mathrm{N}}\right)}{v\left(\overline{\mathrm{S} 5_{\mathrm{N}}}\right)}$ & $\left.\frac{v\left(\overline{\mathrm{S} 5_{\mathrm{VA}}}\right)-v\left(\mathrm{~S} 5_{\mathrm{N}}\right)}{v\left(\overline{\mathrm{S}} 5_{\mathrm{VA}}\right.}\right)$ \\
\hline 54 & 67 & 70 & 83 & 71 & 15 & 1 \\
42 & 190 & 108067 & 190567 & 108067 & 43 & 0 \\
28 & 230 & 56053 & 221090 & 67053 & 74 & 16 \\
5 & 309 & 114 & 315 & 151 & 63 & 24 \\
404 & 100 & 49 & 96 & 49 & 48 & 0 \\
408 & 200 & 3082 & 5188 & 3094 & 40 & 0.3 \\
412 & 300 & 16102 & 31323 & 23580 & 48 & 51 \\
503 & 143 & 9096 & 12637 & 9601 & 28 & 17 \\
505 & 240 & 13100 & 22236 & 15803 & 41 & 40 \\
507 & 311 & 15137 & 27361 & 25278 & 44 & 25 \\
509 & 348 & 19125 & 36394 & 25684 & 47 &
\end{tabular}

Figure 1: Integrality gaps for small size SPOT5 instances

\begin{tabular}{c|c|c|c|c|c|c} 
Instance & $\begin{array}{c}\text { number of } \\
\text { images }\end{array}$ & $\hat{v}\left(\mathrm{~S} 5_{\mathrm{N}}\right)$ & $v\left(\overline{\mathrm{S} 5_{\mathrm{N}}}\right)$ & $v\left(\overline{\mathrm{S} 55_{\mathrm{VA}}}\right)$ & $\frac{v\left(\overline{\mathrm{S} 5_{\mathrm{N}}}\right)-v\left(\mathrm{~S} 5_{\mathrm{N}}\right)}{v\left(\overline{\mathrm{S} 5_{\mathrm{N}}}\right.} \%$ & $\frac{v\left(\overline{\mathrm{S} 5_{\mathrm{VA}}}\right)-v\left(\mathrm{~S} 5_{\mathrm{N}}\right)}{v\left(\overline{\mathrm{S} 5_{\mathrm{VA}}}\right)} \%$ \\
\hline 1401 & 488 & 176056 & 300000 & 188370 & 41 & 6 \\
1403 & 665 & 176140 & 300149 & 188467 & 41 & 6 \\
1405 & 855 & 176179 & 300207 & 188551 & 41 & 6 \\
1502 & 209 & 61158 & 64160 & 64155 & 4 & 4 \\
1504 & 605 & 124243 & 191279 & 139585 & 35 & 11 \\
1506 & 940 & 168247 & 276863 & 183978 & 39 & 8
\end{tabular}

Figure 2: Integrality gaps for big size SPOT5 instances

or $j$ before $i$. Thus, we are not able to define $G_{\mathcal{E}}^{c}$ from the instance description. Consequently, we propose to construct $G_{\mathcal{E}}^{c}$ with the polynomial time algorithm presented in [5] for determining a transitive orientation of a comparability graph. When this algorithm is applied on an arbitrary graph, it defines a circuit-free orientation of edges. Thus, applied to the complementary graph of $G_{\mathcal{I}}^{c}$, this algorithm defines a circuit-free directed graph (transitive when $G_{\mathcal{I}}^{c}$ is a cocomparability graph) that can be used instead of $G_{\mathcal{E}}^{c}$.

For each instance, we compute with Cplex 8.0 the optimal solution value of $\overline{\mathrm{S}}_{\mathrm{N}}$ and $\overline{\mathrm{S} 5 \mathrm{VA}}$. The following tables 1 and 2 present the obtained results.

Upper bounds provided by $\overline{\mathrm{S} 5} \mathrm{VA}_{\mathrm{VA}}$ are logically closer to the optimal solution value of $\mathrm{S} 5_{\mathrm{N}}$ than those provided by $\overline{\mathrm{S} 5_{\mathrm{N}}}$. Let us remark that $G_{\mathcal{I}}^{c}$ is a cocomparability graph only for instances $54,42,1502$ and, in these cases, $v\left(\overline{\mathrm{S} 55_{\mathrm{VA}}}\right)$ is very close to optimal solution 
value. Moreover, upper bounds provided by $\overline{\mathrm{S} 5_{\mathrm{VA}}}$ are more interesting for difficult big size instances with one knapsack constraint $5_{\mathrm{N}}$.

Some improvements would be possible with another instance description. First of all, the algorithm we use to define an orientation of the complementary graph of $G_{\mathcal{I}}^{c}$ remains to select in an arbitrary way a cocomparability sub-graph of $G_{\mathcal{I}}^{c}$; it is obvious that $S 5_{\mathrm{VA}}$ would be strengthened if it is based on the real enchainability graph $G_{\mathcal{E}}^{c}$.

Moreover, $\mathrm{S} 5_{\mathrm{VA}}$ can be strengthened in rewriting contraints $4_{N}$. These constraints are due to the limitation of the instantaneous data flow through the satellite telemetry. A much better formulation is to write cumulative constraints of that type: at each time, the sum of the data flows used by images must be lower or equal to a certain limit.

\section{Conclusion}

The daily satellite mission planning problem can be seen as a stable set problem with additional linear constraints. In this paper, we compare linear relaxations of two 0-1 linear formulations. In the first one, stable set polytope is represented by edge polytope while, in the second one, stable set polytope is exactly described. The theorical difference between these two formulations are also illustrated by numerical experiments on benchmark instances. It is shown that the 0-1 linear programming is of real interest for modeling and solving satellite mission planning.

\section{References}

[1] P. Barcia, J.O. Cerdeira (2003) Node packings on cocomparability graphs, Operations Research Letters 31:341-342

[2] E.Bensanna, G.Verfaillie, J-C.Agnèse, N.Bataille, D.Blumstein (1996) Exact and approximate methods for the daily management of an earth observation satellite, Proc. of the $4^{\text {th }}$ Int. Symposium on Space Mission Operations and Ground Data Systems, Munich, Germany

[3] E. Bensanna, M. Lemaître, G. Verfaillie (1999) Earth Observation Satellite Management, Constraints 4:293-299

[4] V. Gabrel, C. Murat (2003) Mathematical programming for Earth observation satellite mission planning, in Operations Research in Space and Air, T.A. Ciriani, G. Fasano, S. Gliozzi, R. Tadei editors, Kluwer Academics Publishers:103-122 
[5] P.C. Gilmore, A.J. Hoffman (1964) A characterization of comparability graphs and of interval graphs, Can. Journal. Math. 16:539-548

[6] M. Grötschel, L.Lovàsz, A. Schrijver (1988) Geometric Algorithms and Combinatorial Optimization, Springer-Verlag

[7] A. Pnueli, A. Lempel, S. Even (1971) Transitive orientation of graphs and identification of permutation graphs, Can. Journal. Math. 23(1):160-175

[8] M. Vasquez, J.-K. Hao (2001) "A logic-constrained" knapsack formulation and a tabu algorithm for the daily photograph scheduling of an earth observation satellite, Journal of Computational Optimization and Applications 20:137-157.

[9] M. Vasquez, J.-K. Hao (2003) Upper Bounds for the SPOT5 Daily Photograph Scheduling Problem, Journal of Combinatorial Optimization 7:87-103 